\title{
The Rapid Estimation of the Area under the Thermo-Dilution Gurve
}

\author{
Kohji Tamura, M.D., Yutaka Arai, M.D., \\ and Hiroshi Murooka, M.D.
}

\section{Summary}

(1) The forward triangle method vs. the semilogarithmic method showed the regression equation of $\mathrm{Y}=0.211 \mathrm{X}-171\left(\mathrm{~mm}^{2}\right)(\mathbf{r}=0.897)$.

(2) The integral method vs. the semilogarithmic method showed the regression equation of $\mathrm{Y}=1.021 \mathrm{X}-104(\mathrm{r}=0.994)$.

(3) The tangential method vs. the semilogarithmic method showed the regression equation of $\mathrm{Y}=1.006 \mathrm{X}-210(\mathrm{r}=0.992)$.

These data showed that these 3 methods for the rapid estimation of the area under the thermodilution curve were applicable practically, with the reasonable accuracy, the simplicity and the cost.

\section{Additional Indexing Words :}

Thermodilution method Forward triangle method Integral method Tangential method

T order to measure the cardiac output by the indicator dilution method, it is basic to compute the area under the indicator dilution curve after the Stewart-Hamilton relationship by using either the semilogarithmic paper or the planimeter. This method, however, time consuming. Therefore, in the dye dilution method, the various simplified methods for the rapid estimation of the area have been developed for this purpose. ${ }^{1-5)}$

In the thermodilution method, by using the benefits of the methodology itself, ${ }^{11)}$ the similar methods for the rapid estimation were examined. ${ }^{61-10}$ These methods have made the thermodilution to be more easy and practical clinically.

The objects of this study were the followings: (1) whether the forward triangle method is applicable as in the dye dilution method, and (2) whether the electrical integrator is applicable for the measurement of the rapid estimation in a short time and accurately.

\section{Objects and Method}

The thermistor catheter of F5 (Webster Lab., USA) was inserted into the

From the First Department of Medicine, Niigata University School of Medicine, Niigata.

Received for publication April 2, 1973. 
pulmonary artery under the fluoroscopy of the anesthetized closed chest adult canine heart. A 7F Lehman catheter was inserted into the right atrium for the injection of the thermal indicator. The thermal indicator of the $5 \%$ glucose in water $(5 \mathrm{ml}$ of the $2^{\circ}$ to $4^{\circ} \mathrm{C}$ ) was injected manually in a single bolus. The injection was repeated in every $1 \mathrm{~min}$. The indicator in the catheter after the injection was quickly withdrawn rapidly so that the indicator in the dead space in the catheter was not left. The estimation of the cardiac output was carried out as reported already. ${ }^{11), 12)}$ In order to increase the cardiac output, the arteriovenous fistula was made between the femoral artery and vein. On the contrary, the decrease of the cardiac output was caused by letting out the blood.

i) The semilogarithmic method (Stewart-Hamilton method):

The theoretical area of the curve was measured after the Stewart-Hamilton method by plotting on the semilogarithmic paper. This theoretical area was compared with the following 3 methods for the rapid estimation.

ii) Forward-triangle method:

The forward triangle method calculates the area of the initial portion of the curve by multiplying the build-up time and the peak concentration and dividing by 2 (Fig. 1).

iii) The integral method:

Since the recirculation of the indicator distorts the primary curve on the exponential decay, the integrated area is valid only before the appearance of the recirculation, while the rest of the area has to be corrected of the recirculation and added to the first part of the area. Based on this principle and on a simple mathematical function, Sanborn computer (Model 130) has been used for the dye dilution method. ${ }^{5)}$ It is a fundamental exponential property that the value of the integral

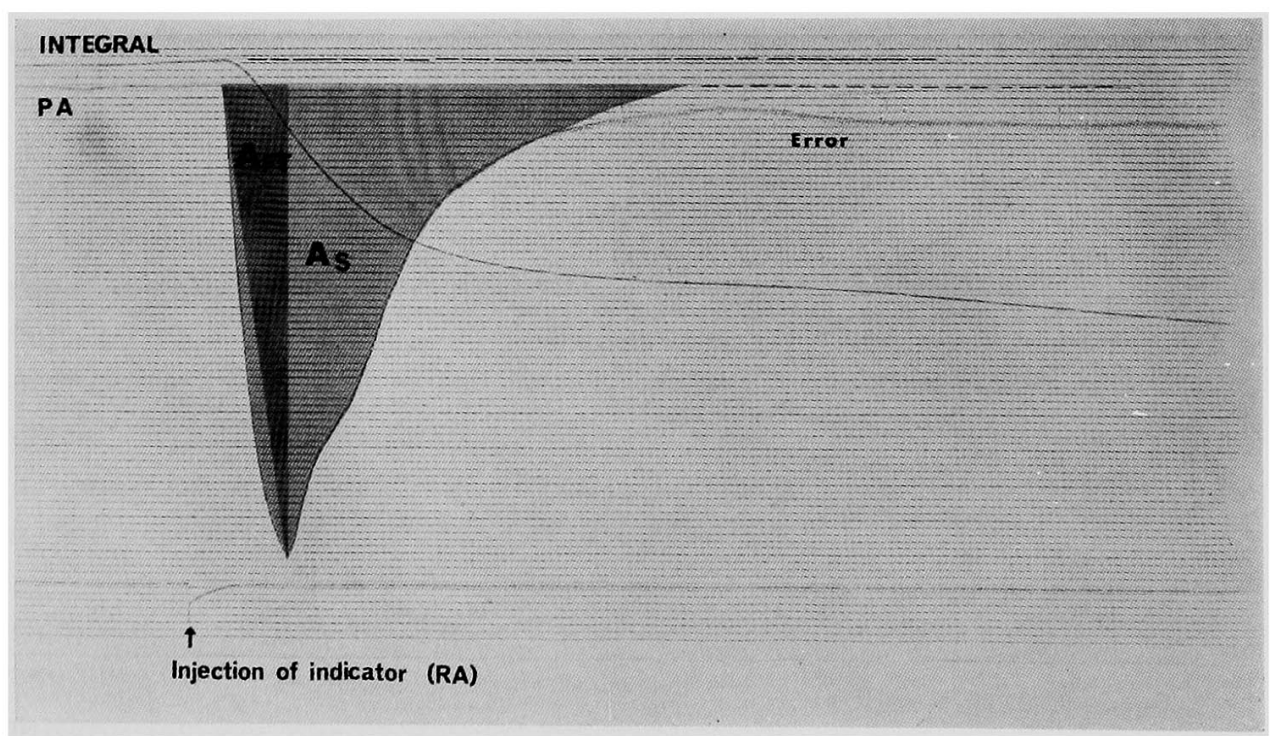

Fig. 1. The comparison of the measurement of the area under the thermodilution curve by the forward triangle method against the semilogarithmic method.

INTEGRAL $=$ the curve of the electrical integration, $P A=$ pulmonary artery, $\mathrm{RA}=$ right atrium, $\mathrm{As}=$ the calculated area by the semilogarithmic method, AFT $=$ the area calculated by the forward triangle method. 


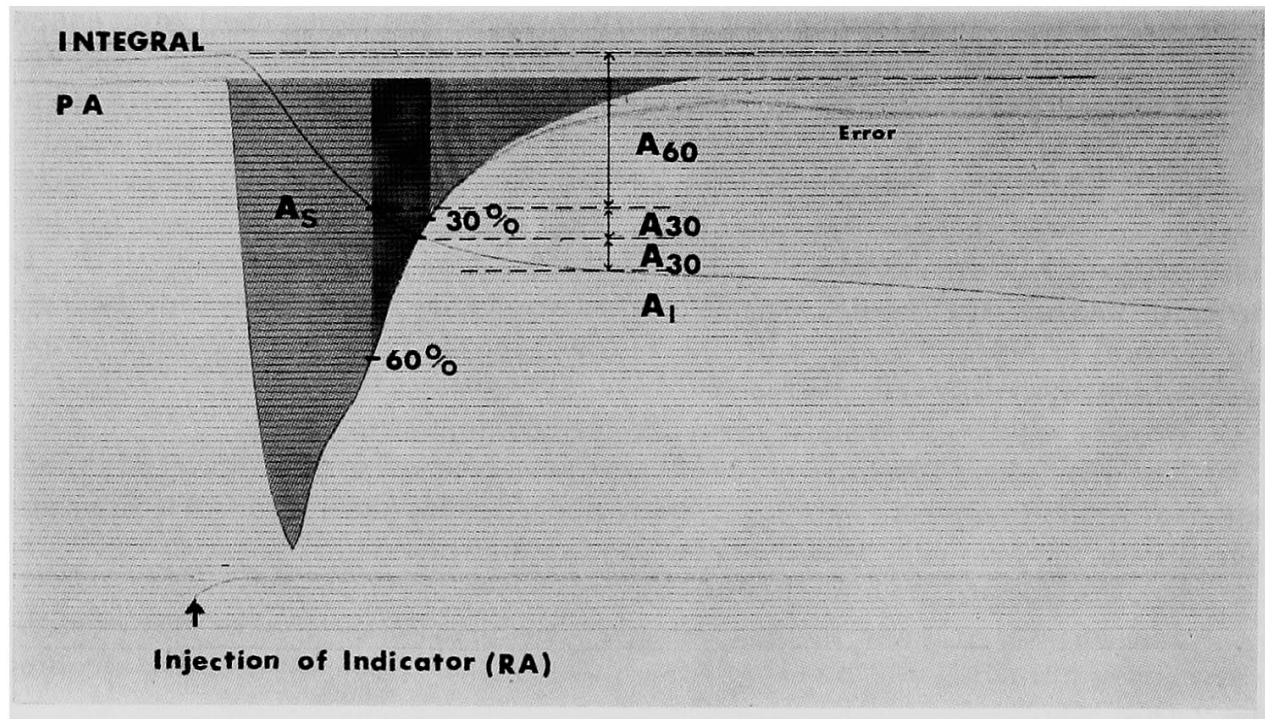

Fig. 2. The comparison of the measurement of the area under the thermodilution curve by the integral method against the semilogarithmic method. $\mathrm{As}=$ the calculated area by the semilogarithmic method, $\mathrm{A}_{\mathrm{B0}}=$ the area until the $60 \%$ deflection of the peak concentration, $A_{30}=$ the area between $60 \%$ and $30 \%$ deflection of the peak concentration, $A_{I}=$ the area by the integral method $\left(A_{I}=A_{60}+A_{30} \times 2\right)$.

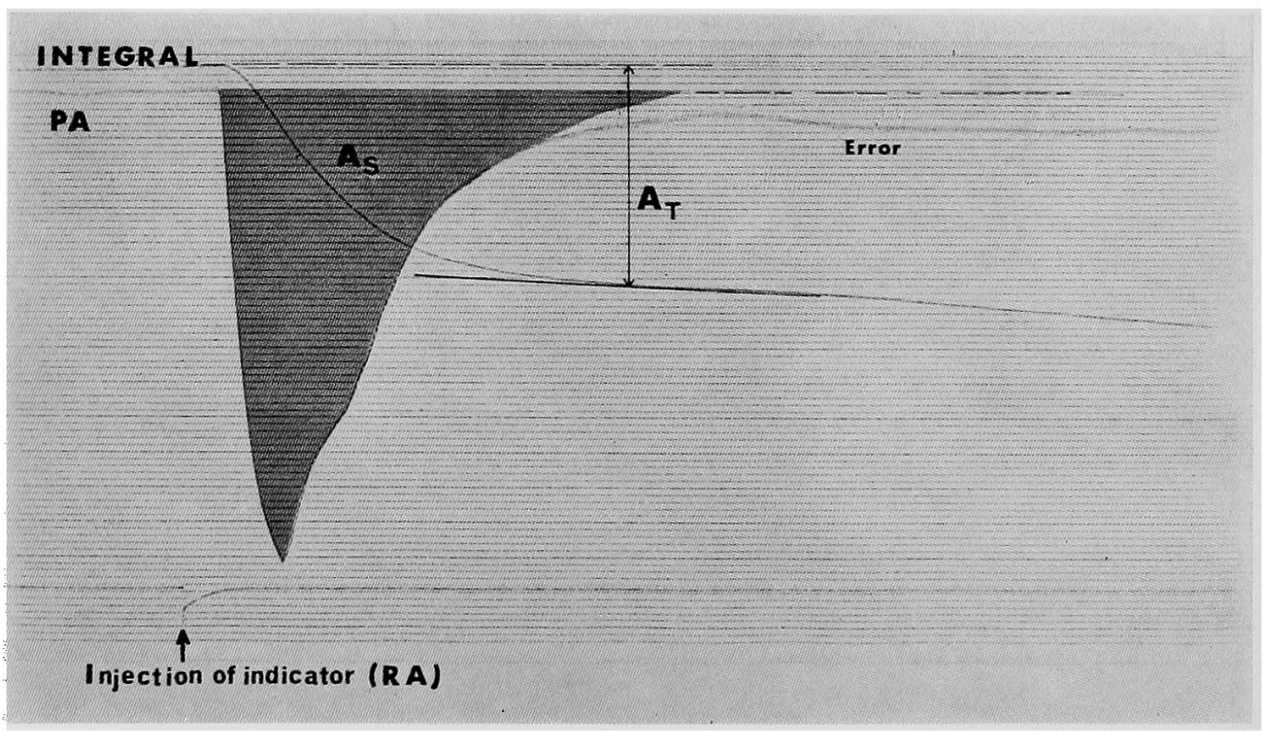

Fig. 3. The comparison of the measurement of the area under the thermodilution curve by the tangential method against the semilogarithmic method.

As = the area by the semilogarithmic method, $A_{T}=$ the area by the tangential method. 
between 2 ordinates of the known ratio is directly proportional to the integral from the first ordinate to infinity.

Therefore, in our experiment, this principle was applied (Fig. 2). This principle was the simple multiplication. Namely the total integrated area $\left(A_{1}\right)$ equals to the area under the thermodilution curve downslope until $60 \%$ of the peak deflection $\left(A_{60}\right)$ and the addition of the 2 times of the area between 60 and $30 \%$ of the deflection $\left(A_{\mathbf{3 0}}\right)$. The calibration of the integral curve was easily performed by adding the known electrical resistance to one arm of the bridge circuit.

iv) The tangential method:

The thermodilution curve (Fig. 3) becomes to show the constant deflection at the terminal portion of the exponential decay after the deflection of less than $10 \%$ of the peak. Therefore, as shown in Fig. 3, the electrically integrated curve became almost the the straight line. The measure scale was put along this straight line and the crossing point was marked. The deflection to this mark was calculated by the comparison to the calibration. This area was thought to be almost similar to the theoretical area.

\section{Results}

i) The forward triangle method vs. the semilogarithmic method:

In the Fig. 1, the indicator was injected at the point of the arrow into the right atrium (RA) and the thermistor in the pulmonary artery (PA) showed the thermodilution curve. The theoretical area under the curve (As) was shown to be the gray area. The primary curve of the dilution shows the occurrence of the error on the exponential decay and the curve never returns to the original base line.

The forward triangle method calculated the area as shown to be the dark area $\left(A_{F}\right)$.

As shown in Fig. 4, the areas were compared 28 times in the 2 dogs simultaneouly by these 2 methods. The result was plotted by putting the data of the semilogarithmic method on the horizontal line against the data of the forward triangle on the vertical line. The scale of the vertical line was magnified 4 times against the horizontal line. The regression equation was $\mathrm{Y}=0.211 \mathrm{X}+171\left(\mathrm{~mm}^{2}\right)$ and the correlation coefficient was 0.994 .

ii) The integral method vs. the semilogarithemic method:

As shown in Fig. 2, the primary curve was electrically integrated. The comparison of these methods was summarized in the Fig. 5. The data of the semilogarithmic method was plottd on the horizontal line and the data of the integral method was plotted on the vertical line against the semilogarithmic method. The areas were compared 27 times in 2 dogs. The regression equation was $\mathrm{Y}=1.021 \mathrm{X}-104\left(\mathrm{~mm}^{2}\right)$ and the correlation coefficient was 0.994 .

iii) The tangential method vs. the semilogarithmic method:

The result was shown in Fig. 6. The areas were compared 25 times 


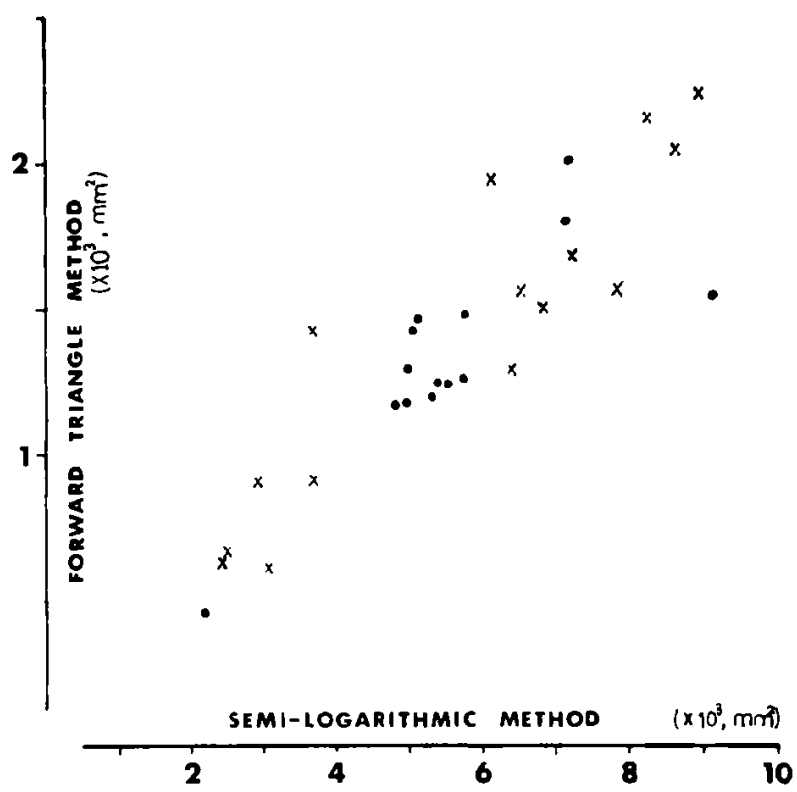

Fig. 4. The data of the comparison of the forward triangle method against the semilogarithmic method. The difference of the marks in the figure shows the difference of the dog.

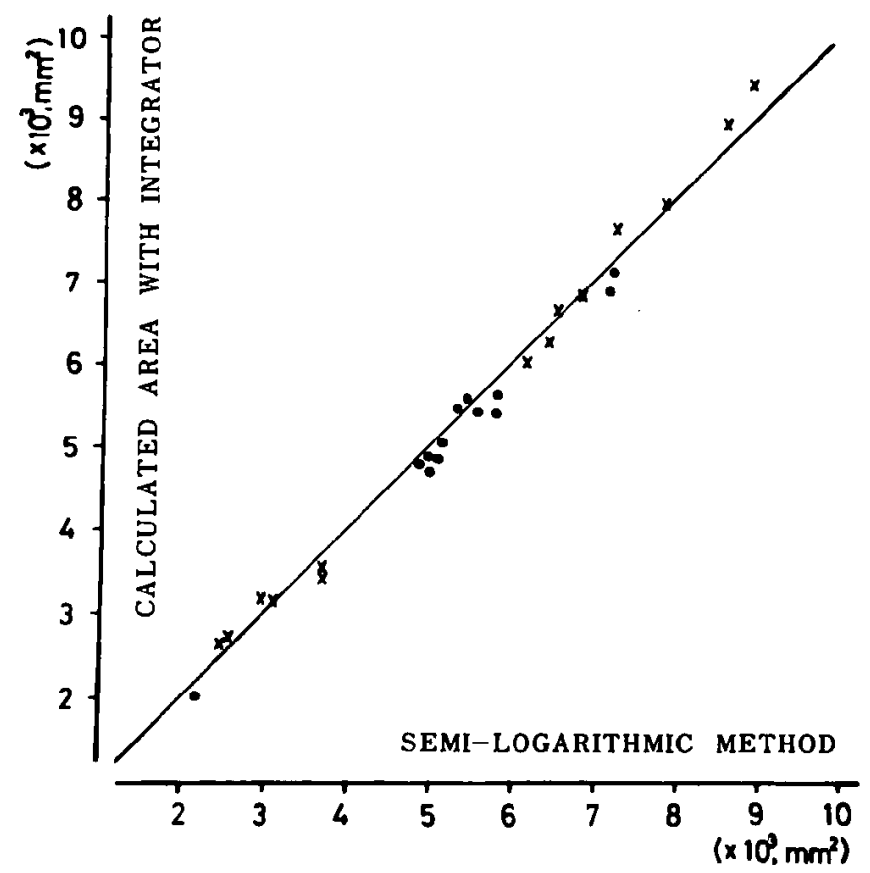

Fig. 5. The data of the comparison of the integral method against the semilogarithmic method. The difference of the marks in the figure shows the difference of the dog. 


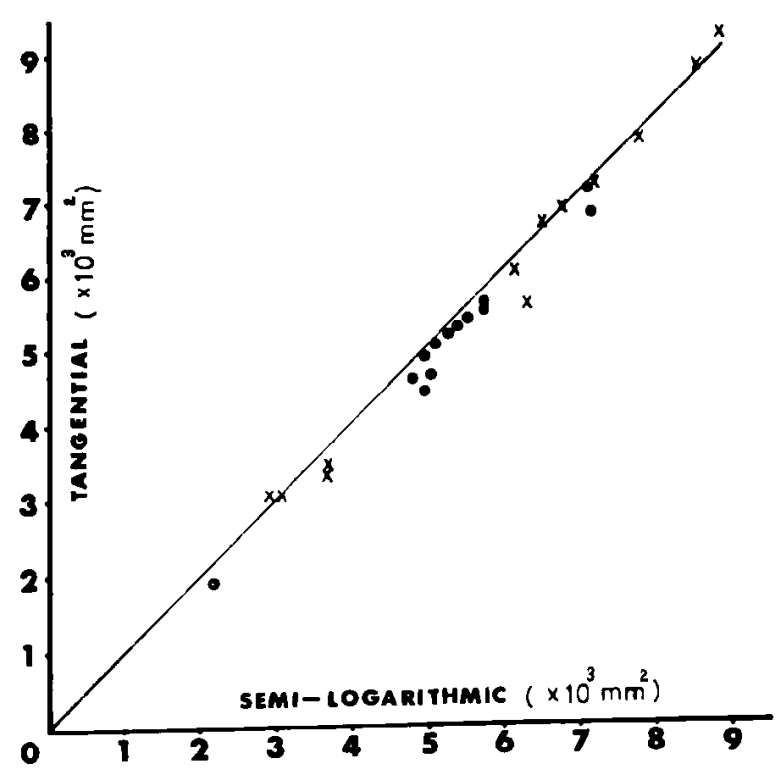

Fig. 6. The data of the comparison of the tangential method against the semilogarithmic method. The diffrence of the marks in the figure shows the difference of the dog.

in 2 dogs changing the cardiac output. The regression equation was $\mathrm{Y}=$ $1.006 \mathrm{X}-210\left(\mathrm{~mm}^{2}\right)$ and the correlation coefficient was 0.992 .

\section{Discussion}

The methods for the rapid estimation of the area under the dye dilution curve have been well examined already.1)-5) In the dye dilution method, the primary curve distorts on somewhere on the exponential dacay, even though the distortion depends on the distance from the injection site of the indicator to the sampling site. Therefore, the correction should be performed to avoid this error. On the contrast to this dye dilution method, the thermodilution curve shows the less distortion of the primary curve because of the practical disappearance of the recirculation of the indicator. ${ }^{11), 12)}$ The thermodilution method, however, is relatively new in the clinical medicine. ${ }^{11)}$ Even though it has the several advantages to measure the cardiac output over the other methods used so far, it is essential to devise the simple technique for the performance and the calculation. ${ }^{8)}$

The forward triangle method in the dye dilution method has been popular and the comparison study with the semilogarithmic method showed the error of $10.42 \pm 8.52 \%$ or $0.52 \pm 0.43 \mathrm{~L} / \mathrm{min}^{2}{ }^{2)}$ In the thermodilution method, the 
similar comparison showed the correction factor of $0.250^{71}$ and the factor was quite similar to our data of 0.211 .

The integral method of Sanborn computer showed, in the dye dilution method, the comparison study with the semilogarithmic method of the regression equation of $\mathrm{Y}=11.09 \times$ Sanborn $-0.301(\mathrm{r}=0.985)$. Pavek ${ }^{9}$ utilized this Sanborn principle in the similar dog experiment to ours and reported the correlation coefficient of 0.998 with the variability of $3.7 \%$ (standard deviation of the difference in per cent of the mean value) and without the systemic deviation. Slama ${ }^{10)}$ also showed that the distortion by the recirculation was ruled out stopping the integration at the two thirds of the peak deflection. He utilized the following formula: $\left(F_{1}(2 / 3 \mathrm{max})+F_{2}\right) /\left(F_{1}\right)=1.72$ (where, $F_{1}=$ the integrated area until $2 / 3$ deflection of the peak concentration and $F_{2}=$ the rest of the area).

Our data also showed the integral method is practically applicable and the cost of the apparatus is very low compared to the usage of the analog computer.

Sanmarco ${ }^{6}$ used the analog computer (automatic base line circuit, integrator, divider and 3 calibration potentiometer) and the integration was stopped at the point of the $10 \%$ deflection of the peak. The comparison of this method and the electromagnetic flow meter of the aortic cardiac output showed the mean deviation of less than 10\%. Our method is similar practically to this Sanmarco's method, because the straight line was taken and the crossing point of the line was practically located less than $10 \%$ of the peak. However, we took the straight line because the measurement is simple and $10 \%$ line still showed the unstable line in most cases. Our data of the comparison with the semilogarithmic method showed the good agreement and this method was the most simple proceduce.

\section{ACKNOWLEDGEMENT}

The authors are greatly indebted to Prof. M. Matsuoka for the preparation of the manuscript.

\section{REFERENCES}

1. Williams JCP, O'donovan TPB, Wood EH: J Appl Physiol 21: 695, 1966

2. Benchmol A, Dimond G, Calvalho FR, Roberts MW: Am J Cardiol 12: 119, 1963

3. Hetzel PS, Swan HJC, Ramidez de Arellano AA, Wood EH: J Appl Physiol 13: 92, 1958

4. Oriol A, McGregor M: Am J Cardiol 20: 826, 1967

5. Taylor SH, Macdonald HR, Sapru RP: Brit Heart J 29: 352, 1967

6. Sanmarco ME, Phillis CM, Marquez LA, Davila JC: Am J Cardiol 28: 54, 1971

7. Kochsiek K, Kleinsorg H, Schütz RM: Z Kreislaufforsch 51 : 346, 1962 
8. Ganz W, Donoso R, Marcus HS, Forrester JS, Swan HJC: Am J Cardiol 27: 392, 1971

9. Pavek K, Karvic K: Z Kreislaufforsch 58: 1109,1969

10. Slama H, Piiper J: Z Kreislaufforsch 53: 322, 1963

11. Tamura K: Shinzo 3: 443, 1971 (in Japanese)

12. Tamura K et al: Shinzo 3: 609, 1971 (in Japanese) 